1

$\mathrm{HW}--30401-\mathrm{VOl} \cdot 5$

DE 33011239

13is

$3 x^{2}$

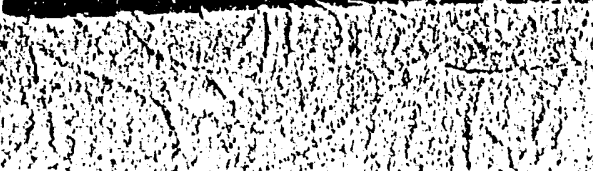

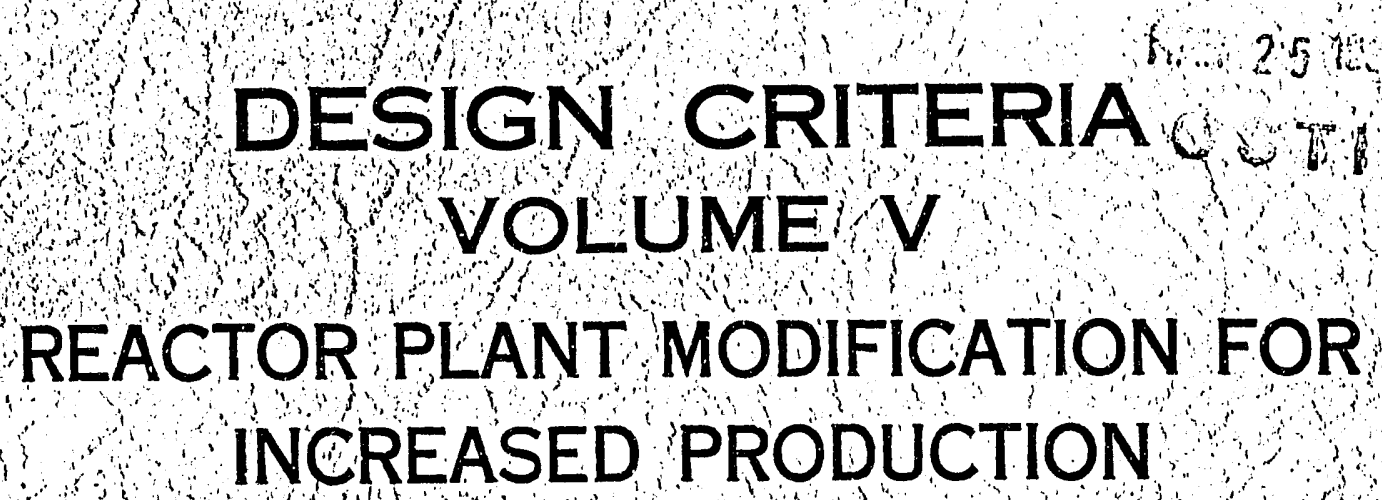

AND

100-C AREA ALTERATIONS

WPROJECT CG-558 AND PROJECT CG-600

(3)

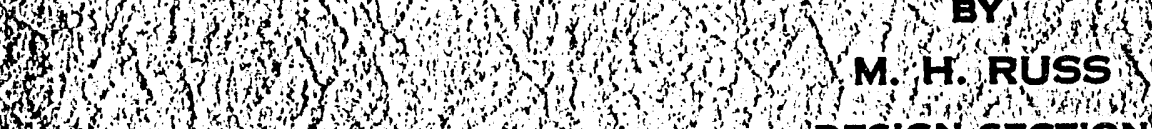

W
S

HANFORD ATOMIC PRODUCTSOPERATION

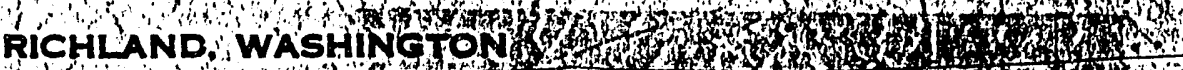
STENERAL
ELCPACG 
VOLUME V

DESIGN CR ITERIA

REACTOR PLANT MODIFICATIONS FOR IICREABSD PRODUCTIOA AND

100-C AREA ALTRRATIONS

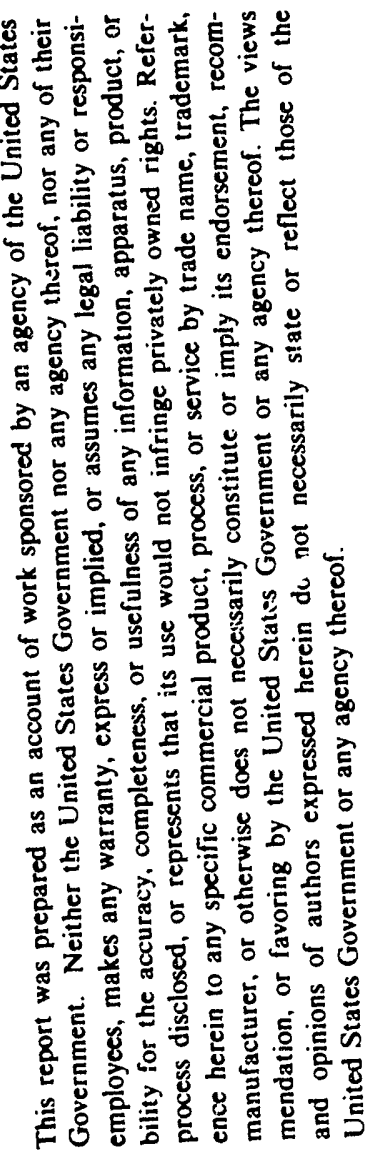

\author{
PROJECT CG-558 \\ AND \\ PROJECT CQ-600
}

\title{
SECTION F
}

CODES, STANDARDS AND BIBLIOGRAPEY

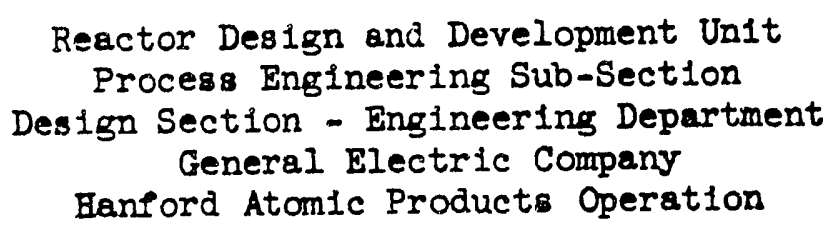


W. K. Alexander

R. K. And̄ersen

G. H. Bauer

R. H. Beaton

C. R. Bergdah 1

J. I. Boyd

$\mathrm{H}, \mathrm{A}$. Carlberg

A. B. Carson

E. S, Day

W. J. Dowis

W. F. Garetson

O. H. Greager

A. B. Greninger

I. O. Hasselblad

T. W. Hauff

H. W. Heacock/E. R. Rudock

J. M. Heffner

H. H. Hubble

E. M. Johnston

C. E. Kent

J. P. Langan

W. K. MacCready

J. C. McLaughlin

J. S. McMahon

J. E. Maider

R. O. Mehann

DNE. P. Peabody

O. H. Pilkey

P. H. Reinker

R. B. Richards

W. D. Richmond

M. H. Russ

H. P. Shaw

J. M. Smith, Ir.

F. A. R. Stainken

J. I. Thomas

J. H. Warren

J. C. Wood

W. K. Woods 300 File

Yellow File

Extra 

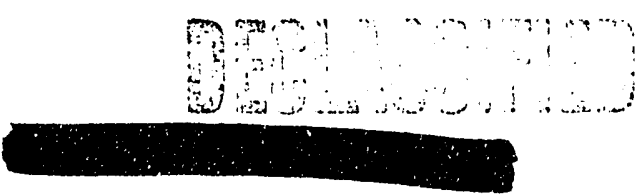

$\mathrm{Hiv}-30401 \mathrm{~V} 5$ Page 3

DESIGN CRITERIA

$\mathrm{K}^{\text {I }}$ CIOR PLAN' MODIFICATION - HROJECT CG-550

AND

100-C AREA AITERATTIONS - PROJECT CG-600

TABLE OF CONTENTS

SECTION F

CODES, STANDARDS AND BIBLIOGRAPHY

Page

I. CODES AND STANDARDS

II. BIBLIOGRAPHY

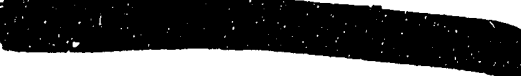


VOLUME $\mathrm{V}$

DESIGN CRITERIA - REACTOR PLANT MODIFICATION

PROJECT CG-558

SECTION F
CODES, STANDARDS, AND BIBLIOGRAPHY

I. CODES AND STANDARDS

Unless otherwise indicated within these Design Criteria, the design, the material to be used, and the work to be performed shall adhere to the requirements set forth in the latest edition of Hanford Works Standards, Design Guides and approved codes.

II. BIBLIOGRAPHY AS OF MAY 1, 1955

\begin{tabular}{|c|c|c|c|}
\hline Doc. No. & Title & Author & Date \\
\hline HAN -51439 & $\begin{array}{l}\text { Reactor Plant Modification for } \\
\text { Increased Production }\end{array}$ & DF Shaw & $8-6-53$ \\
\hline HW-27778 & $\begin{array}{l}\text { Maximum Capacities of the } 100-B \\
\text { Water Plant }\end{array}$ & No Strand & $4-27-53$ \\
\hline$H W-28137$ & $\begin{array}{l}\text { Maximum Capacities of the } 100-F \\
\text { Water Plant }\end{array}$ & No Strand & $5-22-53$ \\
\hline $\mathrm{HW}-28360$ & $\begin{array}{l}\text { Maximum Capacities of the 100-D, } \\
\text { DR Water Plants }\end{array}$ & NO Strand & $6-12-53$ \\
\hline HW-28378 & $\begin{array}{l}\text { Minutes of Meeting No. I - Joint } \\
\text { Meeting of Research and Develop- } \\
\text { ment Committee RDS-10 and RDS-11, } \\
\text { Development Program for Modif1- } \\
\text { cations of Existing Reactors }\end{array}$ & JR Wolcott & $6-12-53$ \\
\hline $\mathrm{HW}-28390$ & $\begin{array}{l}\text { Permissible Pressures in } 190-105 \\
\text { Process Piping at } B, D, F, D R \text { and } H\end{array}$ & WJ Love & $6-30-53$ \\
\hline$H W-28425$ & $\begin{array}{l}\text { Permissible Water Flow Rates to } \\
\text { 105-C }\end{array}$ & GM Roy & $6-18-53$ \\
\hline $\mathrm{HW}-28426$ & Water Plant Expansion - Technical & RB Richards & $6-22-53$ \\
\hline HW-28444 & $\begin{array}{l}\text { Expansion of Production Capacity - } \\
\text { 100-H }\end{array}$ & GM Roy & $6-19-53$ \\
\hline $\mathrm{HW}-28543$ & $\begin{array}{l}\text { Project Proposal. Reactor Plant } \\
\text { Modification for Increased Produc- } \\
\text { tion }\end{array}$ & CE Kent & $6-29-53$ \\
\hline $\mathrm{BW}-28548$ & $\begin{array}{l}\text { P1le Technology Development Test } \\
\text { Number DT-105-524-E } 100 \text { Areas Filter } \\
\text { Plant Hydraulic Limit Tests }\end{array}$ & WCA Woods & $6-29-53$ \\
\hline
\end{tabular}


Doc. No.

HW-28558

HW-28563

$\mathrm{HW}-28594$

HW-28657

HW-28698

HW -28716

HW-28739

HW-28983

HW-28982

HW-29022

HW-29138

HW -29238

HW-29281

HW-2935I
Title

Capacity Test of Building 190 Pump Units 100-B \& 100-F Areas

Technical Notes on Protection Against Boiling Damage

Comparison of 100-H Area Annual Opera ting Conditions

Preliminary Evaluation of the Economics for the Replacement of Existing Reactors

Comparison of Current Annual Operating Costs for the 100-B, 100-D, \& 100-F Plants with the Estimated Operating Costs Under the RDS-D-II Water Plant Modification Program

Minutes of Meeting to Establish Basis for Instrumentation Scope Design on Water Plant Modification Program RDS-D-11 for the 100-B and 100-C Plants

Expansion of Water Treatment Facili- WL Pearl ties, 100 Areas

Process Aspects of Reactor Operation RO Mehann with Increased Protection from Panellit

Increased Reactor Safety Through Panellit Gage and Safety Circuit Revisions

Preliminary Study of the Pile Effluent SS Jones Piping System

Program for Use of Venturis and Supplemental Orifices to Increase Production in Hanford Reactors

An Eng, and Economic Evaluation of Proposed Modif. to Existing Reactor Plants RDS-10 and RDS-11

Installation of Supplemental Orifices and Venturis in all Hanford Reactors

Technical Bases for Pile Outlet Temperatures, Project CG-558
Author

Date

JC McLaughlin

GW Wells

LH McEwen

$$
7-1-53
$$

RK Andersen

$7-2-53$

RK Andersen

$8-5-53$

RK Andersen

$7-14-53$

RK Andersen

$7-16-53$

Ro Mehann

$8-10-53$

$7-20-53$

$8-7-53$

$10-20-53$

JE Robb

$9-8-53$

GM Roy

$9-4-53$

JE Maider

$9-15-53$

WCA Woods

9-15-53

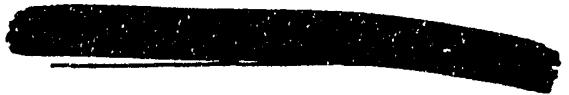


Doc. No.

HW-29352

HW-29353

HW -29367

HW -29374

HW-29399

HW -29419

HW-29457

HW -29484

HW-29527

IIT -29529

HW -29542

HW-29634

$H W-29655$

HW-29707

HW -29709

HW -2971.2
Title

Shielding Aspects of Project CG-558

Technical Bases for Pile Water Flow Rates, Project CG-558

Header Pressure Requirements Using Standard and Enlarged Fittings Project CG-558

A Comparison of Tube Flow-Monitoring

Filter Plant Expansion

An Engineering and Economic Evaluation of Proposed Modifications to Existing Reactor Plants RDS-10 and RDS-II

PT-105-517-E Supplement A, 100 Areas Filter Plants

Process Tube Flow Rates Using Standard and Enlarged Fittings

Reactor Plant Modification Additions to the $230 \mathrm{KV}$ Electrical Transmission Line Facilities

Technical Bases for Evaluation of Production Gains Project CG-558

Project Proposal, Alum Activated Silica Water Treatment Facilities

Graphite Temperature Limit

Biological Shield Operating Conditions at Higher Power Levels

Summary of Alternative Cases for Project $\mathrm{CG}-558$

Alternative Capacity Increases for Project CG-558 - Reactor Plant Modifications for Increased Production

Memo on C-Pile Portion of Project CG558
Author

Date

WK Álexander

$9-15-53$

WK Alexander

WCA Woods

WK Alexander

$9-16-53$

WD Gilbert

RF Recht

WL Pearl

$9-21-53$

JR Wolcott

$9-28-53$

WCA Woods

$10-5-53$

HH Greenfield 10-1-53

WD Gilbert

HR Hughes

$10-2-53$

JR Wolcott

$10-2-53$

CE Kent

$10-19-53$

DH Curtiss

$10-15-53$

CA Pursel

$10-10-53$

VD Nixon

JR Wolcott

$10-21-53$

WE Johnson

$10-21-53$

WK Alexander

$10-22-53$ 
Doc. No.

HW -29768

HW -29778

HW-29813

HW-29822

HW -29932

HW -29934

HW -29970

HW -30065

HW-30118

HW-30205

HW-30283

HW-30379

HW-30401 VI

$\mathrm{HW}-30401$ V2
Title

Test of the Response of an Area Boiler and Process Turbines to a Sudden Increase in Load

Interim Report Pile Technology Development Test 105-524-E 100 Areas Filter Plant Hydraulic Limit Tests

Design Data for Modification of $B$, $D, F, D R$ and $H$ Water Plants

Clearwell Capacity Requirements for 100 Area Modification Program

Justification for C Pile Water Plant Expansion

Minutes of Meeting, C-Pile Flow Rates Project CG-558, Reactor Plant Modifications

An Analysis of the Economics and Hazards Associated with Process Tube Instrumentation

Interim Rpt. No. 1 PT-105-4-MR and PT-105-4-MR Supplement a Evaluation of Poison Column Control Facility

Recommendation for Installation of Back-Up Process Tube Instrumentation

Preliminary Froject Proposal Reactor Plant Modification for Increased Production Project CG-558

Instrumentation for Thermal Shield Cooling Tubes, Project CG-558

Tie Straps for Biological Shields

Design Criteria Volume I Reactor Plant Modifications for Increased Production and 100-C Area Alterations Project CG-558 and Project CG-600

Design Criteria-Reactor Plant Modifications for Increased Production and 100-C Area Alterations Project CT-558 and Project CG-600. Section C, 100-D and 100-DR Plant Modifications.
Author

Date

WJ Love

$11-13-53$

WCA Woods

$11-19-53$

RA Huggins

$11-6-53$

RA Huggins

$11-3-53$

RB Richards

$11-2-53$

JR Wolcott

$11-11-53$

ME Forsman

$11-13-53$

RD Shilling

$9-10-53$

OH Greager

$11-30-53$

HE Hylbak

$2-4-54$

RK Andersen

$12-14-53$ CA Pursel

CC Steele

$12-28-53$

MH Russ

$8-10-54$

MH Russ

$8-10-54$ 


\begin{tabular}{|c|c|c|c|}
\hline Doc. No. & Title & Author & Date \\
\hline $\mathrm{HW}-30401 \mathrm{~V} 3$ & $\begin{array}{l}\text { Design Criteria Reactor Plant Modifi- } \\
\text { cations for Increased Production } \\
\text { Project CG-558 }\end{array}$ & MH Russ & $8-15-54$ \\
\hline $\mathrm{HW}-30401 \mathrm{~V} 4$ & $\begin{array}{l}\text { Design Criteria Volume IV Reactor } \\
\text { Plant Modifications for Increased } \\
\text { Production and 100-C Area Alterations } \\
\text { Project CG-558 and Project CG-600 }\end{array}$ & MH Fuss & $8-15-54$ \\
\hline$H W-30689$ & $\begin{array}{l}\text { Memorandum on Temp Increase in Process } \\
\text { Tubes after Power Failure for Case } \\
\text { 4-A at 100-H \& 100-F Plants }\end{array}$ & MH Russ & $1-29-54$ \\
\hline $\mathrm{HW}-30865$ & $\begin{array}{l}\text { Interim Report, Horizontal Rod Con- } \\
\text { version Development Program - Old } \\
\text { Piles }\end{array}$ & $\mathrm{RL} \mathrm{CaII}$ & $2-26-54$ \\
\hline $\mathrm{HW}-30996$ & $\begin{array}{l}\text { Stability of Synchronous Motors in } \\
100 \text { Area Water Plants }\end{array}$ & $\begin{array}{l}\text { A.J McCrocklin } \\
\text { etal }\end{array}$ & $7-15-54$ \\
\hline $\mathrm{HW}-31084$ & $\begin{array}{l}\text { Project CG- } 558 \text { - Synchronous Motors } \\
\text { for Process Pump Drives }\end{array}$ & $\begin{array}{l}\text { EP Peabody } \\
\text { MH Russ }\end{array}$ & $3-10-54$ \\
\hline $\mathrm{HW}-31162$ & $\begin{array}{l}\text { Procurement of Horizontal Rods - } \\
\text { CG-558 }\end{array}$ & HP Shaw & $3-17-54$ \\
\hline $\mathrm{HW}-31234$ & Project Proposal 100-C Alterations & AA Janos & $5-6-54$ \\
\hline $\mathrm{HW}-31520$ & $\begin{array}{l}\text { Production Gains Resulting from the } \\
\text { Use of the Poison Column Control } \\
\text { Facility on B Reactor, etc. }\end{array}$ & CW Botsford & $4-23-54$ \\
\hline$H W-31689$ & $\begin{array}{l}\text { PT 105-517-E, Suppl. B, } 100 \text { Areas } \\
\text { Filter Plant Tests }\end{array}$ & WCA Woods & $5-10-54$ \\
\hline HW -31938 & $\begin{array}{l}\text { Interim Report PT 105-568-A } \\
\text { Horizontal Rod Skin Temperature } \\
\text { Determination }\end{array}$ & JH Rector & $8-6-54$ \\
\hline HW-32029 & $\begin{array}{l}\text { Cavitation in the Outlet Fittings } \\
\text { at the } B, D, F \text { Piles }\end{array}$ & HH Greenfield & $6-3-54$ \\
\hline$H W-32108$ & $\begin{array}{l}\text { Recommendations Concerning Secondary } \\
\text { Pressure Monitor System, CG-558 }\end{array}$ & HH Greenfield & $6-11-5 i$ \\
\hline $\mathrm{HW}-32113$ & $\begin{array}{l}\text { Project CG-558 - Scope Drawing Trans- } \\
\text { mittal }\end{array}$ & MH Russ & $5-25-54$ \\
\hline $\mathrm{HW}-32168$ & $\begin{array}{l}\text { Additional Justification for Back- } \\
\text { Up Instrumentation }\end{array}$ & MH Russ & $6-18-54$ \\
\hline
\end{tabular}

HW-30401 V5

Page 8

Doc. No.

$\mathrm{HW}-30401 \mathrm{~V} 3$

cations for Increased Production

Project CG-558

Plant Modifications for Increased

Production and 100-C Area Alterations

Project CG-558 and Project CG-600

Tubes after Power Failure for Case

Interim Report, Horizontal Rod Conversion Development Program - 0ld

Stability of Synchronous Motors in

Project CG-558 - Synchronous Motors

for Process Pump Drives

Procurement of Horizontal Rods -

Project Proposal 100-C Alterations

Production Gains Resulting from the Use of the Poison Column Control

PT 105-517-E, Suppl. B, 100 Areas

Interim Report PT 105-568-A

Horizontal Rod Skin Temperature

Cavitation in the outlet Fittings

Recommendations Concerning Secondary

Pressure Monitor System, CG-558

Project CG-558 - Scope Drawing Trans -

Up Instrumentation
Author

Date

$8-15-54$

$8-15-54$

AJ McCrocklin 7-15-54

EP Peabody 3-10-54

$\begin{array}{ll}\text { AA Janos } & 5-6-54 \\ \text { CW Botsford } & 4-23-54\end{array}$

WCA. Woods

$5-10-54$

$8-6-54$

$6-3-54$

$6-11-5$

$5-25-54$

$6-18-54$ 


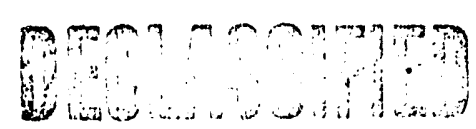

HW-30401 V5 Page 9

Doc. No.

HW -32185

HW-32491

HW -32887

HW -32963

HW -33389

HW-33761

HW -33996

$H W-34312$

$\mathrm{HW}-34354$

HW-35031

HW -35565

HW -35760

\section{Title}

Final Report - Production Test 105524-A Washer Seal and Seal Leakage

Tests for the Horizontal Rod

Investigation of Panellit System Reliability

Reactor Plant Modifications Project (CG-558) Revised During Plans for

"Back-Up" Instrumentation for Reactors

Final Report - Design Test No. 36 Functional Test of 105-K Horizontal Control Rod Prototype Equipment

Project Proposal, Revision 4 Reactor Plant Modifications for Increased

Production (Project CG-558)

3500 HP Process Pump Drives 190-C Operational Limitations

An Improved Method of Process Tube Installation

Front Face Crossheader Check Valves

Strength of Existing Front and Rear Biological Shields when used to Carry Axial Process Tube Loads

Dev. of a Washer Seal for the $B, D$, $F, D R$ and $H$ Conversion Horlzontal Rods

Project CG-600 - 100-C Process Water System Modifications

Pressure Drop-Flow Characteristics of Project CG-558 Inlet Assembly
Author

Date

PM Jackson

$6-22-54$

HH Greenfield 7-20-54

$A B$ Greninger 8-27-54

RL $\mathrm{Call}$

$9-7-54$

HE Hylbak

$12-28-54$

FJ Mollerus

$11-12-54$

GO Amy, et al 12-31-54

AK Hardin

$1-4-55$

RD Schilling

WJ Love

$1-7-55$

PM Jackson

$2-8-55$

MH Russ

$3-2-55$

HH Greenfielã 3-14-55 


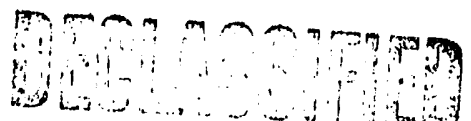

HW-30401 V5 Page 10

\section{PROJECT REPRESENTATIVES MINUTES AS OF MAY 1, 1955}

Minutes No.

1

2

3

4

5

6

7

8

9

10

11

12

13

14

15

16

17

18

19

20

21

22

23

24
Document No.

Undocumented

HW-29964

Undocumented

HW-30191

HW-30273

$\mathrm{HW}-30381$

HW-31112

HW-30900

HW-30901

HW -30776

HW-3.1019

HW-31020

HW-30971

HW-31367

HW-31256

HW-31224

HW -31610

HW - 32668

HW-31879

HW-32007

Undocumented

HW -32660

HW-32817

HWN -32842
Document Date

$9-25-53$

$11-12-53$

$11-23-53$

$12-7-53$

$12-15-53$

$12-28-53$

$1-8-54$

$1-20-54$

$1-25-54$

$2-4-54$

$2-9-54$

$2-1.5-54$

$3-1-54$

$3-4-54$

$3-5-54$

$3-24-54$

$4-13-54$

$5-3-54$

$5-17-54$

$6-2-54$

$6-15-54$

$6-23-54$

$7-2-54$

$7-30-54$ 
Minutes No.

25

26

27

28

29

30

31

32

33

34

35

36

37

38

39

40

41

42
Document No.

HW-32946

HW-3304I

HW-3350I

HW - 33256

HW-33427

HW-33603

HW-33905

HW-33906

HW- 34314

HW-34172

HW-34436

HW-34195

HW-34272

HW-34892

HW-35723

HW-35870

HW -36060
$\mathrm{HW}-30401$ V5 Page 11

Document Date

$8-6-54$

$9-13-54$

$9-20-54$

$9-30-54$

$10-8-54$

$10-19-54$

$10-25-54$

$11-5-54$

$11-16-54$

$12-3-54$

$12-10-54$

$12-17-54$

$12-30-54$

$2-3-55$

$2-17-55$

$3-21-55$

4-1-55 


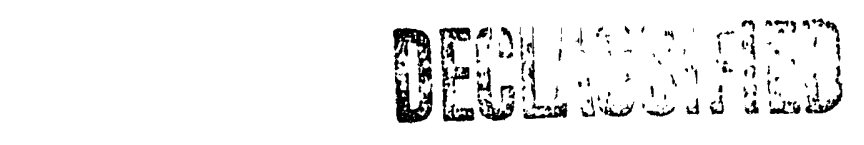

$\mathrm{HW}-30401$ V5 Page 12

PERTINENT DESIGN COUNCIL MINUTES

\section{AS OF MAY I, 1955}

\section{Minutes No.}

91

93

96

97

98

100

101

102

103

204

105

206

107

108

109

110

111

112

114

115

117

128

119

120
Document No.

HW- 29678

HW -30064

HW -30278

HW-30355

HW -30557

HW-30804

HW -30918

HW-31008

HW-31062

HW-31212

HW- 31433

HW -31505

HW-3162O

HW-31792

HW-31921

HW-32130

HW- 32318

EW- 32472

HW'-32790

HW-32895

IIW- -33367

HW-33439

HW-33609

HW-33799
Document Date

10-19-53

$11-23-53$

$12-14-53$

12-23-53

$1-15-54$

$2-8-54$

2-24-54

$3-2-54$

$3-8-54$

$3-25-54$

4-12-54

4-19-54

$4-26-54$

$5-10-54$

$5-24-54$

6-14-54

$7-2-54$

7-19-54

8-18-54

$8-30-54$

10-11-54

10-18-54

$11-1-54$

$11-16-54$ 
Minutes No.

122

123

124

125

127

129

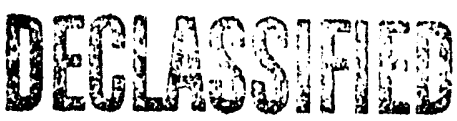

HW-304OI V5 Page 13

Document No.

HW-34189

HW -34383

$H W-34805$

HW-35061

HW- 35718

HW- 36280
Document Date

$12-20-54$

$1-10-55$

$1-27-55$

$2-10-55$

3-10-55

4-18-55 

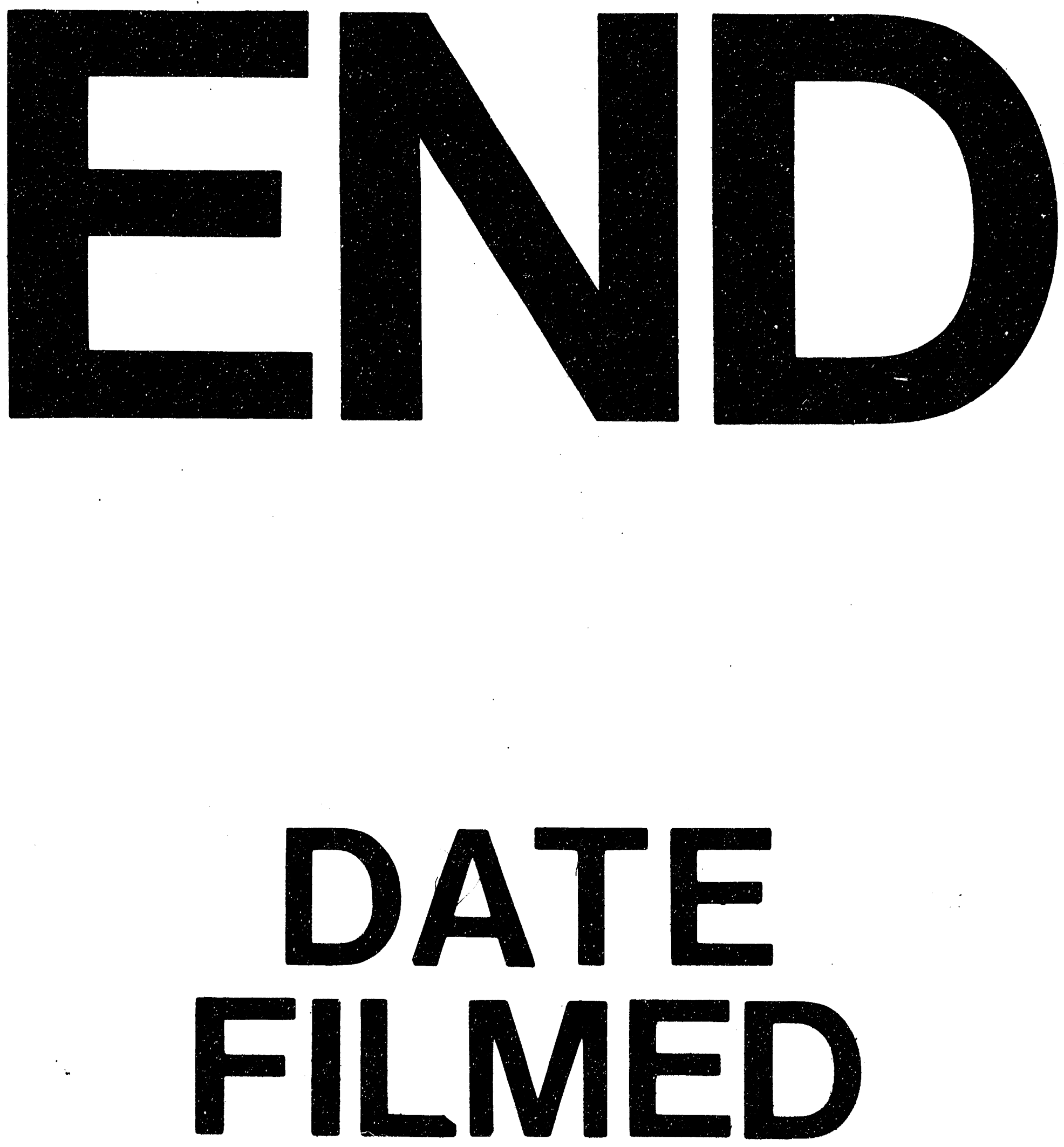

1

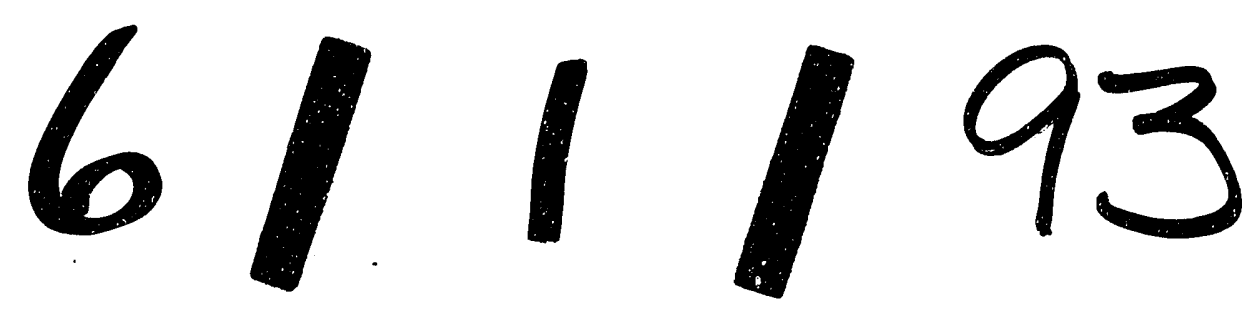


